\title{
Modeling and analysis of the effectiveness of dynamic models of complex automated systems of machine-building production
}

\author{
Andrei A. Cherepashkov ${ }^{1,}$, and Pavel A. Samoylov ${ }^{2}$ \\ ${ }^{1}$ Samara State Technical University (SamSTU), Chair of Machine Building Technology, RF \\ ${ }^{2}$ ASCON-Samara LLC, Samara, RF
}

\begin{abstract}
The article discusses the problem of choosing an efficiency criterion for constructing dynamic models necessary for organizing the management of the design and operation of integrated automated design systems in engineering. The results of comparison of analytical and experimental graphs of integral criterion dynamics in complex projects are presented.
\end{abstract}

\section{Introduction}

In the industrialized countries, the transition to a new economic structure is associated with the massive introduction of information technology. At the same time, the paradigm of the "Digital Economy" affects virtually all aspects of society, including material production. In industry, including in machine building, the process of information technology is manifested in the active creation of complex automated systems at enterprises, the CAD / CAM / CAE ... CA "x" - technologies and systems) and automation tools and product life cycle management integrated into the information environment (CALS / PLM technologies and systems) [1].

The development and implementation of software and methodological complexes and industrial systems are usually carried out by commercial companies (firms integrating automated systems) that specialize in the automation of certain industries [2]. When integrating the automated system created at the enterprise, professional integrators operate with components of the ready-to-use software application (Commercial Of The Shelf COTS) from the leading CAD manufacturers [3]. And also they rely on proven methods of reengineering the design and production processes necessary for the successful implementation and operation of complex software for industrial use.

Over the last years, the world CAD industry has seen significant changes in the methodology for the creation of integrated systems [4]. Increasingly, even in small and

\footnotetext{
*Corresponding author: cher-mail@mail.ru
} 
medium-sized enterprises, they refuse from so-called "boxed" (typical) products in favor of custom engineering. In such adjusted solutions, called "customized" solutions, original or substantially modified application software (bespoke software) [5] is used. To automate the organizational and technical planning and dispatching of machine-building industries, it was primarily not only (and not so much) the functional of universal automated control systems (ERP) that was used, but special software products called MES (Manufacturing Execution System). Moreover, the development and integration of MES in complex systems are performed by specialists of companies specializing in the field of automation of machine-building engineering. As, for example, the system "Gulf Stream" by the leading Russian manufacturer of CAD - the company ASCON [3].

In accordance with the methodology used by integrators for the development and implementation of automated systems, functional models are constructed reflecting various aspects of production and system-wide processes. Traditional approaches recommended by IDEF standards, along with certain advantages (graphical notation, the ability to display not only the sequence of work, but also the mechanisms for their execution, modularity, etc.) [1] have a number of imperfections [2].

Among the problems complicating the practical application of known languages of functional modeling include the complexity of drawing diagrams, the complexity of their reading and analysis, etc. The limitation is the static nature of models in which the dynamics of the development of the system's functioning is not sufficiently fully and clearly reflected. Therefore, practicing specialists often supplement static diagrams of functional models by graphs and diagrams that visualize the development of processes over time. The universal Gantt charts and network graphs [1], used in planning and organization of production, became widely known. For operational management of design and implementation work, specific (subject-oriented) graphics reflecting the dynamics of projects development using a certain criterion characteristic of a particular subject area is also applied. For example, when analyzing the dynamics of the engineering design, the total volume of design documents developed by engineers for the current period of time can be used as the main indicator. Or, graphically, the number of changes made to the set of design documents during the return cycles characteristic of the design of many technical objects is displayed. In this case, the process fits quite accurately into the classical graph of a damped oscillatory process [6].

\section{Practical aspects of using dynamic models to assess the efficiency of computer aided design processes}

Subject-oriented criteria give good results at certain stages of the product life cycle. However, for complex projects involving a number of different stages, local efficiency criteria in dynamic models are not appropriate, and the problem arises with the choice of some integral exponent (indicator) reflecting the process of functioning of an integrated organizational and technical system [2,6].

When designing and deploying complex automated systems for industrial applications (PLM-systems), integrating companies perform a rather complex workflow, including a variety of different stages [1,2]. Starting from the creation of the concept, the development 
of support tools, up to the training of personnel and the pilot operation of the system. Therefore, for an integrated assessment of works on the creation and implementation of automated systems, authors proposed to use such a criterion as the labor intensity of the executed design procedures.

The most obvious and simple for constructing a dynamic model of computer-aided design is a two-dimensional graph, the horizontal axis of which is marked by calendar dates, and on the other axis the total labor intensity of the executed work ( $\boldsymbol{T}$ - spent by all executors for a given period) is postponed (Figure 1, a). The undoubted advantage of such a model is the use of real time (calendar dates and timekeeping of the performers).

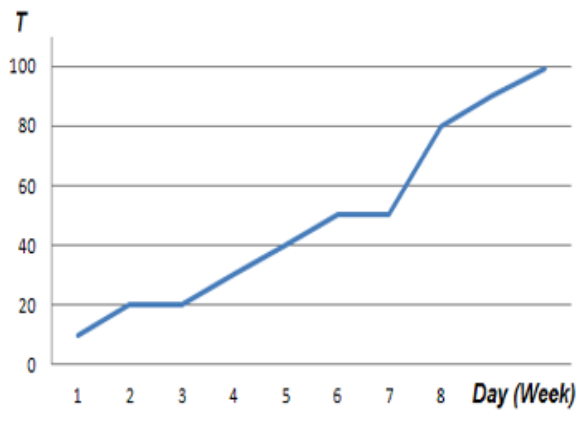

a

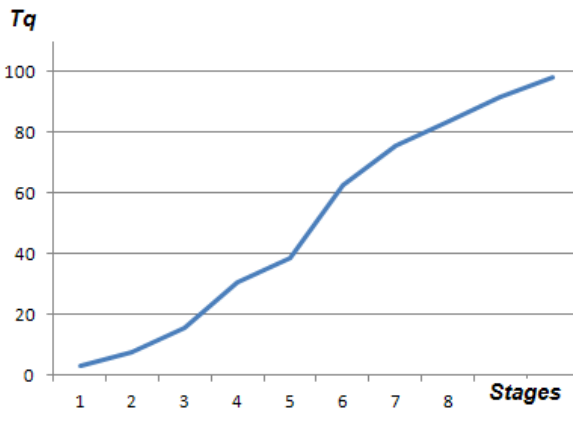

b

Fig. 1. Graphs reflecting the dynamics of design work: a) in real time; b) - in stages, taking into account the effective labor intensity of designing

However, it can be argued that such an approach contains latent uncertainty in the evaluation of the complexity of the various stages, which can differ significantly in complexity and nature of the work. The timing does not take into account the intensity of work, which varies significantly in different stages of the project. In long-term projects, due to organizational, financial and many other reasons, there may be significant time intervals during which the development is partially or even completely stopped. On the contrary, in the project teams there are often enough periods of intensive work, during which a large amount of work is performed in a relatively short calendar period.

It should be specially noted that the time costs fixed by the timing, when performing creative work, which engineering design undoubtedly belongs to, are also not very informative, since they largely depend on the qualifications of the performers.

In the method under discussion, it is proposed to use the expert approach for calculating the integral criterion for the efficiency of engineering works that are difficult to formalize. That is, it is necessary to adjust the real time of execution of works at different stages (or performed by different performers), coefficients that reflect the intensity of work and the qualifications of the performers. Despite the apparent subjectivity in the practice of labor standards, expert assessments are used extremely often and at the same time they give fairly stable and accurate results in a certain professional environment. It is noticed that in the process of long-term activity in the competitive environment of specialized subjects (to which the integrators belong, too), expert estimates of the complexity of standard works are leveled both in absolute and relative terms [2]. In addition, in commercial companies accounting of all paid work is well established. In organizations associated with the 
provision of services, labor intensity are widely used to calculate the cost of work. As a result, the cost of the completed project is estimated by total labor intensity.

In the method of modeling the abscissa proposed by the authors, not calendar dates are marked, but the stages of work (Fig. 1, b); on the ordinate axis, the cumulative total sums the total values of the integral criterion reflecting both the volume of labor produced by the executors, as well as their effectiveness.

$$
T_{q}=\sum_{1}^{n}(t \cdot q)_{i}
$$

where $t$ is the time spent by the performer (timekeeping), $q$ are the experts' assigned coefficients, taking into account the intensity and qualification of the work of specialists participating in the work at the $i$-th stage of the project. Let's call this indicator the effective intensity of the project.

In the idealized case, when it is assumed that all design procedures contribute equally to the formation of the system, the design path must be linear, as shown in the graph in Fig. 2a. On the graph, $T q$ is normalized as a percentage of the total labor intensity of the project.
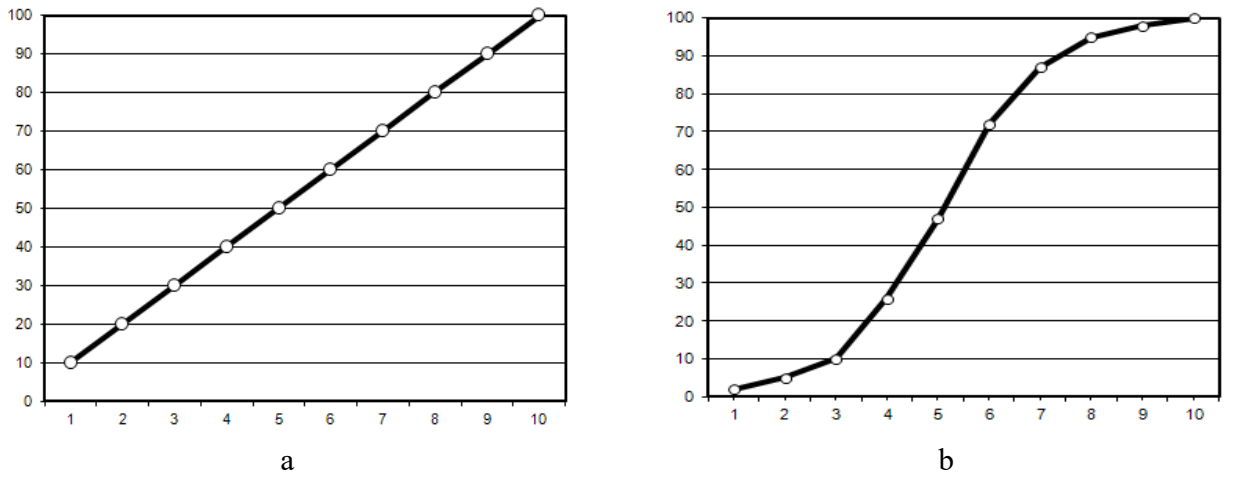

Fig. 2. Theoretical graphs of the dynamics of design works: a) linear graph; b) an idealized "S" shaped curve

The study of the dynamics of the real developments of complex systems development [2] shows that the effective labor intensity essentially varies at different stages of design work. At the initial stages of business process reengineering, an enterprise survey is performed, including well-formalized procedures for collecting statistical information, constructing functional models, etc., which do not require material intensity and highly skilled labor. At the final stages of design, the effective contribution to the development of the project is also slowed down, as the resources of the designers are mainly spent on routine procedures for processing previously adopted design decisions. This explains the convexity ("S" - shapedness) of the trajectory of the development of a complex project. In Fig. 2b, the "reference" design path is presented, which can be obtained if all the work planned by the plan is met regularly and with a certain high quality averaging $(q=1)$.

The construction of dynamic trajectories convincingly demonstrates the main regularities of the implementation projects development. With the help of semi-graphical modeling of certain aspects of the company's activities, it becomes possible to detect 
accurately and quickly the bottlenecks in management, optimize the overall business scheme. Proximity to the reference curve can serve as an integral criterion for the success (efficiency) of the implementation project development. In turn, irregularities in the smoothness or deviation of the project trajectories from the planned ones indicate that there are problems [6].

\section{Selecting and using analytical charts}

In practice, the discussed functional dependence is given in tabular way. However, for idealized (reference) "S" -shaped curves, analytical analogues can be selected, including among classical functions. For example, "S" -shaped curves closely correspond to the graph of the inverse trigonometric function.

$$
Y=\operatorname{arctg}(x)
$$

This function is multivalued. The graph of the principal value corresponding to $Y \subseteq[0$, $\pi]$, which inflection point passes through the origin of coordinates, while the function itself increases monotonically, and then decreases symmetrically in the range $-\infty<x<+\infty$. Through the main value of the function, other similar functions can be expressed, having achieved the necessary task of identifying the correspondence (Figure 3).

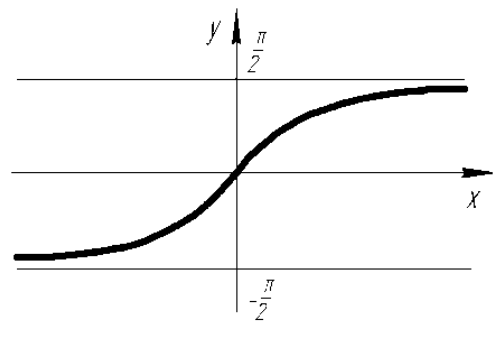

a

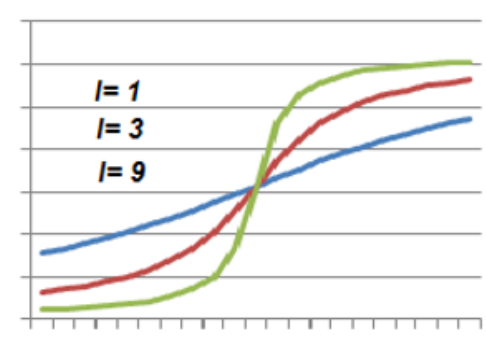

$\mathrm{b}$

Fig. 3. Example of analytical analogues of "S" -shaped curves: a) graph of the principal value of the function; b) change in the bending intensity of the graph using the coefficients

The analytical graphs used in the article used the following relation, bounded by the interval at which the function is monotonic:

$$
Y=\operatorname{arctg}(x \cdot l)+k \cdot \pi,
$$

where the coefficient $k$ allows you to shift the graph to the upper right quadrant so that all real values of the function are positive, and the coefficient 1 allows you to adjust the intensity of the bend. In the graph of Fig. $3 . b$ shows an example of the values $-1<x<+1$;

$l \subseteq\{1,3,9\}$.

\section{Overall results and conclusions}

Fig. 4 shows the application of the analytical "S" curve to real graphs reflecting the development dynamics of the process of creation the development and implementation of 
PLM systems in two characteristic projects of ASCON-Samara [2,6], implemented at industrial enterprises in the Samara region.

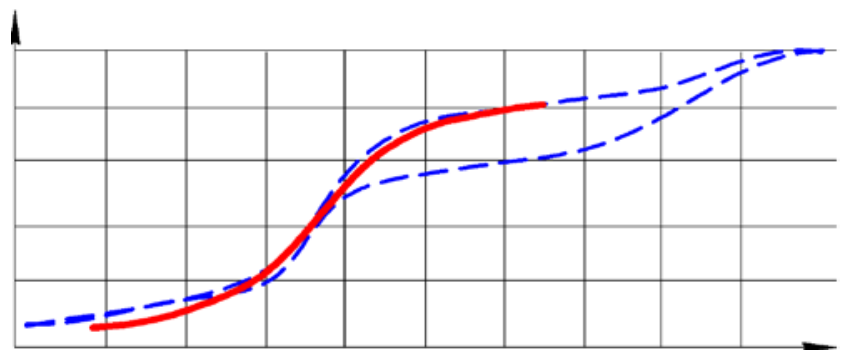

Fig. 4. Analytical curve (solid line), superimposed on real graphs of the effective labor intensity of the process of development and implementation of the PLM system (dotted lines)

In its central part, the analytical curve fits very accurately into the schedule for optimal execution of the project. The second curve shows a deviation from the optimal trajectory, which required the introduction of changes in the program of work (management of the control), which ultimately led to the improvement of the key indicators of the project.

This work was supported by the Russian Foundation for Basic Research (project 18-08-00168).

\section{References}

1. A.A. Cherepashkov, N.V. Nosov, Computer technologies, modeling and automated systems in machine building: textbook (In-folio, 2009)

2. A.A. Cherepashkov, P.A. Samoilov, Problems of development and implementation complex solutions for automation of technical training of machine-building production, Computer integration of production and IPI-technologies: mater. Vseros. scientificpractical. Conf., Orenburg: OSU, p. 118-121 (2017)

3. ASCON [Electronic resource]: Complex solutions for machine building / Company "ASCON". - [Russia]. - URL: http://ascon.ru/ press / news / items /? News = 2043 $(10 / 10 / 17)$.

4. Transition metrics and revenue metrics. Offer alternative scenarios for Autodesk's fiscal year. - [USA]. - URL: http:/gfxspeak.com/2016/02/26/autodesk-revenue-downin-fourth-quarter-flat-for-fiscal-year/ (10.10.17).

5. Bespoke Vs COTS [Electronic resource]: Blueberry Systems, Ltd. - [UK] .- URL: http://www.blueberrysystems.co.uk/Resources/WhitePapers/BespokeVsCommercialOf f-The-Shelf.aspx (10.10.17).

6. A.A. Cherepashkov, P.A. Samoylov, Application of graphoanalytical models in problems of analysis of the processes of development and implementation of complex automated systems, Models, systems, networks in economics, technology, nature and society, Penza, No. 2 (22), p. 268 - 278 (2017) 